\title{
Application of Spectrogram Analysis in Traditional Vocal Music Teaching and Multimedia Animation Vocal Music Teaching
}

\author{
https://doi.org/10.3991/ijet.v11i11.6242 \\ Lin-li Liu ${ }^{1}$, Yi Pang ${ }^{1}$ and Zhuang-li $\mathrm{Hu}^{2}$ \\ ${ }^{1}$ Hunan Institute of Science and Technology, Hunan, China \\ ${ }^{2}$ Wuhan University, Wuhan, China
}

\begin{abstract}
The spectrogram analysis technology via computer application makes voice visualized, realizes the integrated teaching mode of "mouth to ear- nose", and improves the teaching quality of vocal music. The technology gives a dynamic map of singing respiration and singing organs, and the singing process is specified by the harmony search algorithm. This technology solves problems of shallow breathing, slurred speech and incorrect use of resonant cavity. The spectrogram analysis visualizes vocal music teaching, improves students' abilities of singing, practice and innovation.
\end{abstract}

Index Terms - traditional vocal music teaching, multimedia animation vocal music teaching, spectrogram analysis

\section{INTRODUCTION}

With the rapid development of the art of vocal music in China, vocal music teaching quality has also aroused the attention of the relevant departments. Spectrum analysis is an important part in the teaching of vocal music. How to improve the overall quality of the teaching has become a major issue faced by many vocal music teaching [1-2].

New technology is changing the way of our work and study profoundly[3-4], however, at present, in respect to the manner of education research in China is still in its infancy, because want to explore the way of education, first of all, to be well informed about the multiple education methods, to further explore the correct conclusion. Ministry of education on the education facing the 21sl century revitalization action plan [5], the article has made clear that are widely used in the modern information technology in education leads to profound changes in the education system. In terms of vocal music education field, the category of longitudinal fine development and integration with other subjects is an important trend of the development of the contemporary vocal music, increasing its total knowledge information geometric type [6-7]. Reflected in the vocal music discipline, mainly to enrich the song theory, a new mechanism and singing techniques and style of the deepening of the study, with the integration of related subjects and infiltration is increasingly strengthened [8].

In the traditional vocal pedagogy, the one to one face to face teaching method, is also a vocal music teacher to different students take "teach students in accordance with their aptitude" teaching mode. In the present teaching mode, using the spectrum analysis technology, the computer application is used in the teaching process, so that the voice of visualization, realize the comprehensive "mouth to ear- nose" teaching mode.

\section{Method AND ALGORITHM}

The innovation of teaching has been applied in the vocal music classroom teaching implementation, college of art media gathered the vocal music quality education resources, efforts to improve the quality of teaching, make students get the best education. Course construction mainly focus on the larger the range of internal existing classic teaching theory on the basis of using the modem high-tech multi-media teaching of vocal music teaching sharing activities, combined with the advantage of college, break the traditional "dictate syncretism" abstract vocal music teaching mode, the singing teaching in the process of breathing, resonance, articulation, take the lead in using the spectrogram analysis, and animation on spectrogram analysis, visually rich, and as far as possible, improve the traditional vocal music teaching in the abstract, is not easy to master will sing. A new teaching way in the current vocal music teaching and research is an innovation at home and abroad. The vocal music construction will meet the purpose of the cultivation of high-quality talents.

About innovation "spectrogram analysis techniques of singing respiration and singing organs plane dynamic map", M spectrogram analysis animation technology produced by different acoustic area (high, medium and low) singing vocal organs spectrogram analysis dynamic map", and audio and video comprehensive application of network technology related teaching content design and production, is mainly composed of the remote and continue education college Li Jian teacher as the guidance and supervision.

The spectrogram analysis vocalists teaching research, teaching achievement belongs to "the Project of Vocal Music Construction" (Fig.1.) characteristic teaching methods and means of teaching. The results of teaching for free and open online sound related audio and video teaching resources link has played a strong supporting role, realizes the high quality teaching resources sharing. Completely changed in the past of vocal music teachers' subjective understanding on singing organs inaccurate interpretation, solved the teachers for singing organs use description, solved the students understand the deviation caused by the misunderstanding can sing muscle operation out of control; For interdisciplinary teaching and research on the thinking and ideas, methods and means of updating and breakthrough innovation significance to effective development, promote the quality education resources sharing at the same time, will make the students get the 
best education, so as to comprehensively improve the quality of vocal music teaching.

Harmony Search Algorithm (HS) is a new intelligent algorithm which is proposed by Geem $\mathrm{Z}$ W for spectrogram analysis. The algorithm simulates the process in which the musicians repeatedly adjust the pitches of various musical instruments until the musical instruments generate a wonderful harmony. Similar to the process, the $i$ th $(i=1,2, \ldots, m)$ decision variable is to be considered as the $i$ th musical instrument, and the $j$ th $(j=1,2, \ldots, m)$ solution vector in the optimization is to be regarded as the $j$ th harmony generated by the instrument. The objective function of optimization is the evaluation of harmony. There are 3 important parameters involved in HS, i.e., Harmony Memory Considering Rate (HMCR), Pitch Adjusting Rate (PAR), and Band Width (BW).

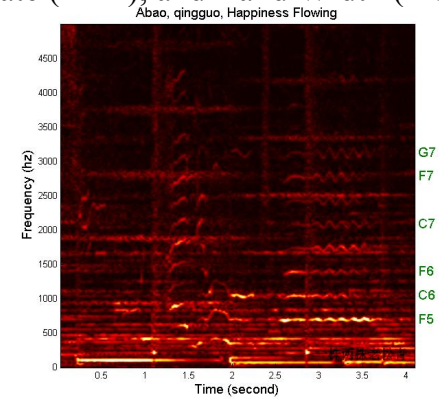

Figure 1. An spectrogram analysis sample

The basic steps of Harmony Search Algorithm are as follows:

Step 1: Setting of the basic parameters

We have to give some value for these parameters, such as the variable number $N$, the range for each variable, the maximum iterations $I_{\max }$, the harmony memory size $S_{H M}, H M C R, P A R$ and $B W$.

Step 2: Harmony memory initialization

$S_{H M}$ solution vectors are randomly generated according to the variable range for each variable. Namely,

$$
H M V=\left[\begin{array}{c|c}
\boldsymbol{x}^{1} & f\left(\boldsymbol{x}^{1}\right) \\
\boldsymbol{x}^{2} & f\left(\boldsymbol{x}^{2}\right) \\
\mathrm{M} & \mathrm{M} \\
\boldsymbol{x}_{H M}^{S_{H M}} & f\left(\boldsymbol{x}^{S_{H M}}\right)
\end{array}\right]=\left[\begin{array}{cccc|c}
x_{1}^{1} & x_{2}^{1} & \mathrm{~L} & x_{N}^{1} & f\left(\boldsymbol{x}^{1}\right) \\
x_{1}^{2} & x_{2}^{2} & \mathrm{~L} & x_{N}^{2} & f\left(\boldsymbol{x}^{2}\right) \\
\mathrm{M} & \mathrm{M} & \mathrm{M} & \mathrm{M} & \mathrm{M} \\
x_{1}^{S_{H M}} & x_{2}^{S_{H M}} & \mathrm{~L} & x_{N}^{S_{H M}} & f\left(\boldsymbol{x}^{S_{H M}}\right)
\end{array}\right]
$$

Step 3: Generating new solutions

In this step, we should create a new harmony $\boldsymbol{x}^{\text {new }}=\left[x_{1}^{\text {new }}, x_{2}^{\text {new }}, \ldots, x_{N}^{\text {new }}\right] . x_{i}^{\text {new }}(i=1,2, \ldots, N)$ can be generated by 3 ways, i.e., learning the harmony memory, tone trim, and random selecting. Hence, we have:

$$
x_{i}^{\text {new }}=\left\{\begin{array}{l}
x_{i}^{j}, j \in\left\{1,2, \ldots, S_{H M}\right\}, \text { if } \text { rand }<H M C R \\
\bar{x}_{i} \in X_{i}, \text { otherwise }
\end{array}\right.
$$

where $X_{i}$ is the range of $x_{i}$ and rand is a random number which belongs to $[0,1]$. As for $x_{i}^{\text {new }}$ from harmony memory, we have

$$
x_{i}^{\text {new }}= \begin{cases}x_{i}^{\text {new }}+\text { rand }^{*} B W, & \text { if rand }<P A R(\text { Continuous }) \\ (k+\lambda) * x_{i}^{\text {new }}, \lambda \in[-1,1], & \text { if rand }<P A R(\text { Discrete }) \\ x_{i}^{\text {new }}, & \text { otherwise }\end{cases}
$$

where $P A R$ is the pitch adjusting rate, $B W$ is band width.

Step 4: Updating the harmony memory

In this step, we should evaluate the new harmony. If the new harmony is better than the worst harmony in the memory, the worst harmony is replaced with the new harmony, i.e.,

$$
\boldsymbol{x}^{\text {worst }}=\boldsymbol{x}^{\text {new }} \text {, if } f\left(\boldsymbol{x}^{\text {new }}\right)<f\left(\boldsymbol{x}^{\text {worst }}\right)
$$

Step 5: Checking the iterations

We should check whether the number of iterations is more than $I_{\max }$. If not, then repeat Steps 1-4; otherwise, we should finish the process, and output the result.

In the field of artificial intelligence, a genetic algorithm (GA) is a search heuristic that mimics the process of natural selection. Genetic Algorithm is an adaptive random search method which derives from the genetics and natural selection in biological evolution. GA is first proposed by John Holland in 1970s.

Vocal music teaching is to promote new technology of multimedia animation that they have been used in some college vocal music teaching, and the praises of the experts, colleagues and students. Using multimedia spectrogram analysis, on animation technology, will sing the human physiological organ motion process dynamic innovative made into video (Fig. 2), in combination with the practical training of students in vocal music singing in breath, resonance, articulation and other specific issues, and then based on the right of teachers demonstration (real audio listening), by adopting the production technology of spectrogram analysis mode to respiratory plane dynamic video and 3 on spectrogram analysis techniques of different acoustic area (high, medium and low) singing vocal organs three-dimensional dynamic video "on the image of the combination of theory and practice of teaching, achieve the personalized targeted teaching problem solving.
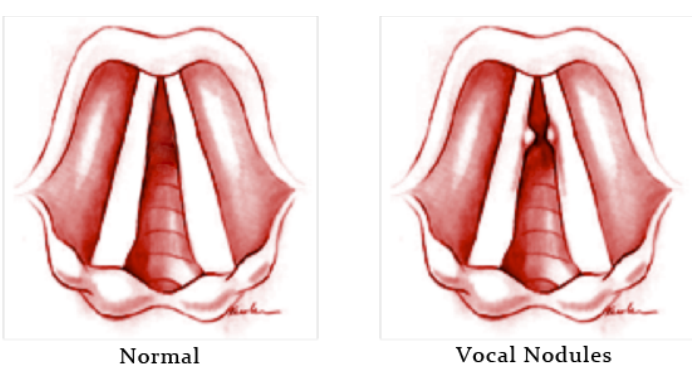

Figure 2. Video of human physiological organ motion process dynamic innovative

\section{EXPERIMENT RESULT}

In order to evaluate the effectiveness of spectrogram analysis in traditional music teaching, the quality of singing after using this method is evaluating. The intuitive understanding of unable to sing the perspective of the human body organ, scientifically correct use in singing performance, specific solved the singing breath shallow, larynx, enunciation unclear, resonance cavity using inaccurate and range extension is difficult problem, make the vocal music, teaching analysis and study on the compre- 
hensive art effect promotion got higher aesthetic requirements and the teaching effect.

Through a detailed analysis of the two methods above, is not hard to find in the different teaching method: both the traditional dictate syncretism type teaching is mainly dictated by the teacher singing skills, and new technology of multimedia animation vocal music teaching is mainly through $3 \mathrm{~d}$ animation intuitive show the change of the cavity and the position of the suction. For example, in the process of how to speak, the traditional model of the teacher first overview to our voices need to breath, put behind, jaws open, voice callings chest, head cavity, flavor stability and the waist to support you.

Figure 3 shows the experiment data of the spectrogram analysis.

Then, the teacher will through their body language, facial expressions and so on for the students to do demonstration. Multimedia teaching mode is the use of advanced multimedia spectrogram analysis, will sing physiological organs dynamic into video formats for innovative motion process, combining students' actual training in vocal music singing breath, resonance, articulation of specific problems, such as, according to the teacher's right demonstration (real audio listening), by adopting the production technology of S spectrogram analysis "singing respiratory plane dynamic video and spectrogram analysis techniques of different acoustic area (high, medium and low) singing vocal organs three-dimensional dynamic video Mon the image of the combination of theory and practice of teaching, achieve personalized targeted teaching problem solving.

Figure 4 shows a spectrogram analysis of a tenor when he sings a song. Figure 5 shows a spectrogram comparison of a tensor and a soprano when he / she sing a same song in a same place.

Both traditional vocal music teaching mode and new technology of multimedia animation type teaching purpose is in order to make students understand how to use singing skills to complete the work. Adhere to the following teaching concept:

Follow the principle of "according to their aptitude.

Adhere to the principle of inspiring and motivational to build democracy, equality, harmonious teacher-student relationship.

Although, traditional teaching method is relatively popular, with the rapid development of vocal music, as increasing of vocal music lovers, the growing interest in vocal music has displayed the traditional teaching mode is difficult to understand, comprehend disadvantages such as slow, based on the characteristics of two kinds of teaching model, framework, content, form the effect of the content such as contrast, fully embody the new technologies of spectrogram analysis vocal music teaching is more intuitive, the advantage of better understanding, thus draw the conclusion: the vocal music teaching model must be able to get bigger promotion by using new technology of spectrogram analysis, music education will have a new progress.

\section{DISCUSSION}

Results: the results show in vocal music training class, reached the intuitive acoustics rapid voices singing quality requirements; Innovation teaching "Spectrogram analysis

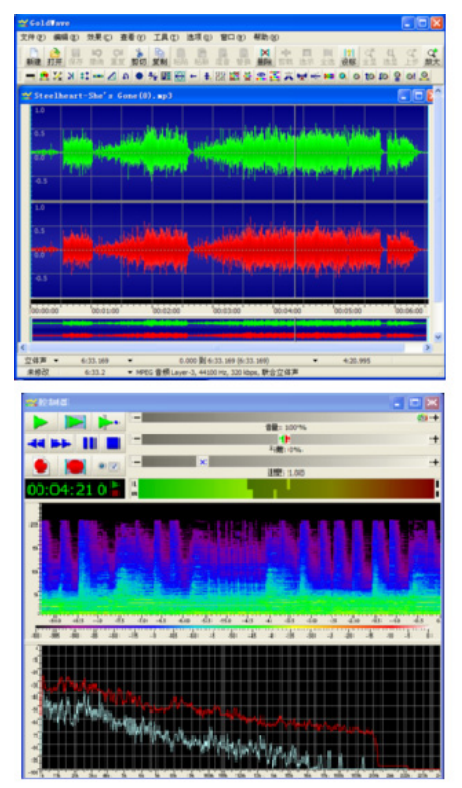

Figure 3. The experiment data of the spectrogram analysis

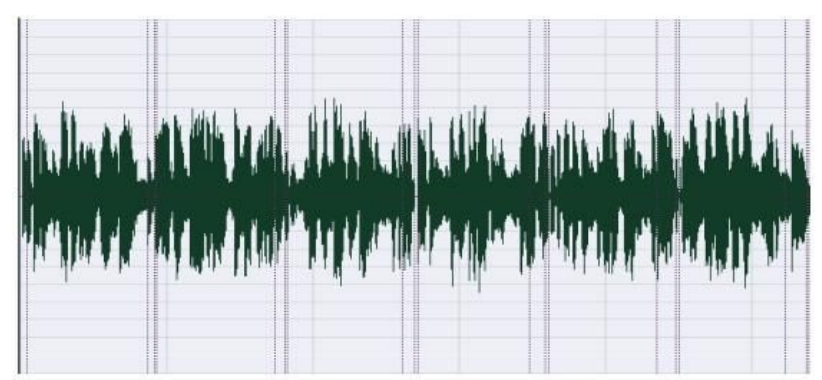

Figure 4. The experiment data of the spectrogram analysis

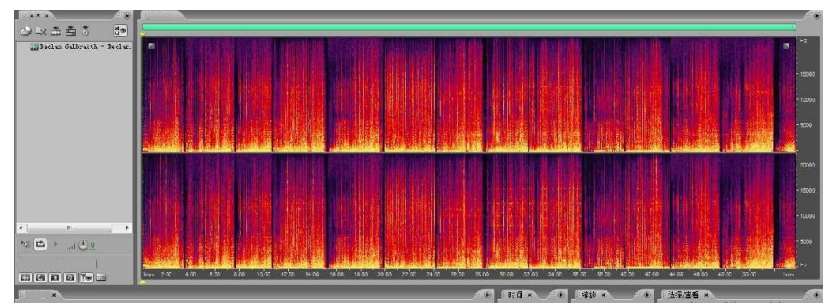

Figure 5. A spectrogram comparison of a tensor and a soprano when he / she sing a same song in a same place

animation - singing respiration dynamic map", spectrogram analysis teaching singing respiratory dynamic map' spectrogram analysis teaching different acoustic area (high, medium and low) singing vocal organs dynamic map", first of all, for vocal music training, classroom teaching provides a fast and efficient to obtain the correct understanding singing organs, accurate manufacture and use effectively "singing instrument" teaching material; The most significant teaching effect, reflect in the final stage of the vocal music, perfect for vocal music art provides scientific teaching support new methods, new means, new technologies, new ways.

Two methods are scientific, specific solve the singing breath shallow, larynx, enunciation unclear, resonance cavity using difficult problems such as inaccurate, range extension. But practice more, make a not learned vocal music students learning vocal music, traditional vocal music teaching method and means is inflexible single, falling behind, there is a lot of limitations, the subject role 
of students in teaching can't get into full play, to improve the students' professional comprehensive quality.

From the result above, we may see Signal/RTS4.0 was used to analyze the sonogram of five high quality bouts for each group. The frequency of high pitch of the tested group was quite high, sometimes reaching or exceeding 5 $\mathrm{kHz}$. Adult males produced boom, pre-modulated note and modulated phrases, whereas adult females produced great frequency of high pitch. Adult males and mated females coordinated to produce complex duets, which usually began with and ended with the male singing. Both male and female singings and their coordinated duets sequences showed obvious differences between the three species. Sonogram characters, based on our results, therefore support the division of these groups of high-pitched voice group into the current three species. We then discussed the possibility of using vocal individuality for population surveys and monitoring population dynamics and dispersal patterns. Significant individuality of vocalization was detected in all 9 males, and the correct rate of individual identification yielded by discriminant function analysis using a subset of variables was satisfactory $(>90 \%)$. Vocal stability over 2-6 years was also documented in 4 males. During the course of this research, we also observed one male replacement (confirmed by vocal analysis). This time-and labor-saving method might be the most effective way to improve the performance in traditional vocal music teaching.

In the teaching of vocal music, the sound can be visualized by scientific instruments, so that the vocal music teaching can be transformed from the traditional mode into the teaching mode of "mouth to eyes to ears".

In traditional vocal music teaching, the physical structure of the vocal organs and body active when teaching content, such as most of the pictures and oral teaching method is expounded, and the students also may only know a little about singing outside. Sing for some details and occurs when the body internal activity status can only be imagined, without reference to visibility, therefore, in the process of the student to practice it will be difficult to fully in accordance with the requirements of the teacher explained to doing it correctly. Especially in the teaching of singing open and concentration and waist abdomen confrontation, deep breath and high position of some key, difficult and complex singing skills, although the teacher speak very detailed, but the student is still difficult to know why. Vocal music teaching method, by contrast, the new technology of multimedia animation is more easy to let students accept, intuitive feel the change in the cavity, under the guidance of the teacher, can quickly find the abdomen, diaphragmatic muscle, chest, laryngeal cavity, head of several important position of the cavity, cavity correct control of breath can see inside the cavity, at the same time also can intuitive see in dealing with the bass, tenor and soprano at three different tone breath and processing of the cavity, the introduction to rapidly and realize fast.

\section{CONCLUSION}

The purpose of this study is to research the application of spectrogram analysis in traditional vocal music teaching and multimedia animation vocal music teaching. The vocal music teaching method and the vocal music teaching method reform is the important guarantee to improve the quality of vocal music teaching, is to cultivate students' innovative spirit and practice ability and learning ability of effective measures. A new means of teaching methods to reform can give full play to the initiative of both teachers and students, enthusiasm and creativity, created the "teaching" and "learning" the interaction of atmosphere, make the abstract visualization of the vocal music teaching, visualization, promoted the students singing ability, practical ability, innovation ability enhancement. And, of course, should be constantly in the analysis, validation, summarized in vocal music teaching, to achieve perfect gradually.

\section{REFERENCES}

[1] Y. Wang, A. Qi, and F. Cui, "Application of the Multimedia Teaching System Based on Real-time Shooting and Production in Martial Art Course," International Journal of Emerging Technologies in Learning, vol. 11, no.3, pp. 37-41, March 2016. https://doi.org/10.3991/ijet.v11i03.5347

[2] J. Zhang, "On the Role of the Vocal Teacher's Capability of Singing Played at Vocal Music Teaching," Canadian Social Science, vol.4, no.2, pp.96-101, 2008.

[3] H. Jing, "The Study on the Impact of Data Storage from Accounting Information Processing Procedure," International Journal of Database Theory and Application, vol. 8, no.3, pp. 323-332, June 2015. https://doi.org/10.14257/ijdta.2015.8.3.28

[4] Z. Lv, J. Chirivella, and P. Gagliardo, "Bigdata Oriented Multimedia Mobile Health Applications," Journal of medical systems, vol.40, no.5,pp. 1-10, May 2016. https://doi.org/10.1007/s10916016-0475-8

[5] C. Bithell, "Polyphonic voices: National identity, world music and the recording of traditional music in Corsica," Ethnomusicology Forum, vol.5, no.1, pp.1324-1329, 1996. https://doi.org/10.1080/ 09681229608567247

[6] J. Zhu, "Multimedia Music Teaching System Application," Key Engineering Materials, vol. 1244, pp.1903-1908,2011. https://doi.org/10.4028/www.scientific.net/KEM.474-476.1903

[7] D. Zbainos, and A Anastasopoulou, "Creativity in Greek Music Curricula and Pedagogy: An Investigation of Greek Music Teachers' Perceptions," Creative Education, vol.03, pp.55-60, 2012. https://doi.org/10.4236/ce.2012.31009

[8] M. Saida, H. Saida, "Application to Vocal Music Training of Newly Developed Strain-Gage Type System for Checking Respiratory Movement in Real Time," The Japan Joumal of Logopedics and Phoniatricst, vol.50, pp. 153-160, 2010. https://doi.org/10.5112/jjlp.50.153

\section{AUTHORS}

Lin-li Liu and Yi Pang are with Hunan Institute of Science and Technology, Hunan, China.

Zhuang-li Hu (corresponding author) is with Wuhan University, Wuhan, China (616100150@qq.com).

The paper is supported by Hunan Province Philosophy Social Science Fund Project Planning (No. 13YBA161). Submitted 09 September 2016. Published as resubmitted by the authors 15 October 2016 . 